Day 1:

Can Our Data Meet the Challenges? 


\title{
The Power of the Unexpected
}

\author{
Brian Warner ${ }^{1,2}$ \\ ${ }^{1}$ Department of Astronomy, University of Cape Town, Cape Town, South Africa \\ ${ }^{2}$ School of Physics and Astronomy, Southampton University, Southampton, UK \\ email: Brian.Warner@uct.ac.za
}

Invited Talk

\begin{abstract}
The history of astronomy has provided variable sources of unexpected kinds-from novae recorded in oriental archives, rapid radio, optical and high-energy changes in white dwarfs, neutron star and black hole binaries, to recent discoveries with satellites. A brief overview is given, as a prelude to a conference that anticipates a tidal wave of observations and discoveries to be made at all wavelengths during the next five to ten years.
\end{abstract}

Keywords. history and philosophy of astronomy, miscellaneous, surveys

\section{Introduction}

This is a conference with many dimensions: one of frequencies or energies from radio to $\mathrm{TeV}$, a range of transients' time-scales from microseconds to decades, recurrence times from milliseconds to tens of years, a range of amplitudes from KEPLER's $10^{-6}$ precision to the most energetic supernovae and gamma ray bursts, and a variety of observational cadences from nanosecond logging of individual photons, future All-Sky better-than hourly coverage, oriental archival records, and even the potential of Antarctic ice cores to record sunspot cycles and supernovae (Motizuki et al. 2009), currently over centuries but perhaps eventually spanning millennia. Some of these are still relatively unexplored parts of phase space but which within a few years will undoubtedly reveal unexpected phenomena.

Many major discoveries in astronomy have derived from chance observations - and many have required the development of new theories for their explanation. As Andy Fabian has emphasised, the role of serendipity in the progress of astronomy can hardly be overestimated (Fabian 2010). And as is the case throughout science, the importance of technological advances is often paramount - new, more sensitive or more accurate instrumentation enables exploration at new wavelengths, energies or time-scales, where unexpected phenomena are lurking. In astronomy at almost all wavelengths the first signs of variability - the theme of this conference - were usually incidental to the main objectives, but often initiated deliberate, more widespread, searches.

\section{Early History}

It was of course known, since the observation of a solar flare by Carrington and Hodgson in 1859, that minor astronomical brightness variations could occur (accompanied, puzzlingly at the time, by strong aurorae and geomagnetic storms), but the earliest examples of major changes had in fact come more than two millennia before with the oriental discoveries of novae (Fig. 1), then largely of prime interest to Emperors' astrologers who wished to keep their heads. 


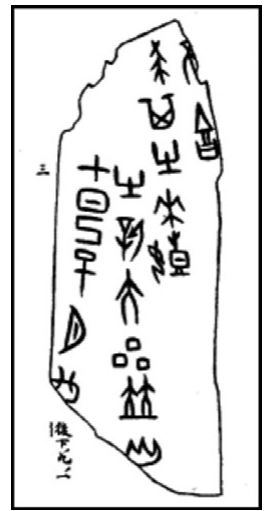

Figure 1. Chinese record of a nova in 1300 BC (Ronan 1994). An early Astrologers' Telegram. From Ronan (1994).

In the West there were very early visual discoveries of periodic variable stars, with Mira in 1596, Algol in 1667 and a total of 10 known by 1786. Only 230 years later, thanks to improvements of detectors and technique, the crop of variable stars we expect from GAIA is about 18 million (Eyer \& Cuypers 2000).

Karl Jansky's announcement in 1932 that the Sun radiates radio waves is a prime example of exploration at a previously untested wavelength, and is the foundation of all radio astronomy, but it was not until 1962 that a strongly variable extrasolar source was recognised (this was the supernova remnant Cas A, first detected at radio wavelengths in 1947). Within the solar system the Sun itself was early known to have variable radio flux, but it is indicative of the then adolescent state of radio astronomy that decametric waves from Jupiter, detected using the most sensitive long wavelength apparatus available to Bernie Burke in 1955 and immediately found to be highly variable, were first reported in the Journal of Geophysical Research (Burke \& Franklin 1955) and only later in the Astronomical Journal (Franklin 1959). This work helped lay foundations for later understanding of the magnetosphere of Jupiter; in particular the modulation of its radio emission by the position of satellite Io in orbit led to a uni- (or homo-) polar inductor model (Goldreich \& Lynden-Bell 1969) with later relevance not only to volcanism on Io but more widely with applications to compact binaries, black holes, pulsars, the moon surrounded by the solar wind, the solar wind itself, sunspots, and planetary magnetic tails. This array of phenomena, illustrating the astrophysical occurrence of the Faraday Disc, making its appearance in a laboratory in 1831, is an example of the universality and wide applicability of basic physics.

Before the 1950s there were only a few indications that the universe contained dynamic events on short time-scales. For example, the term "short period variable star" then usually indicated RR Lyrae or $\delta$ Scuti stars with pulsation periods around 1.5 hours. During the late 1940s the first hints of much more rapid non-periodic luminosity variations appeared - surprisingly from visual observations of cataclysmic variable stars: in recurrent nova T CrB in 1947, in the nova-like variable AE Aqr in 1949, and in VV Pup in 1950. These stars' emergence onto the rapid variability stage included recognition that they are all short period binaries, e.g. UX UMa at 4.7 hours (Linnell 1949) and later ones as short as 1.4 hours, with the implication that at least one of the components must be a white dwarf. This was reinforced by the discovery in 1954 of a 71-second brightness modulation of high stability in the old nova DQ Herculis by Merle Walker (1956), which was of unprecedented shortness, and in retrospect was the first indication of rapid rotation 


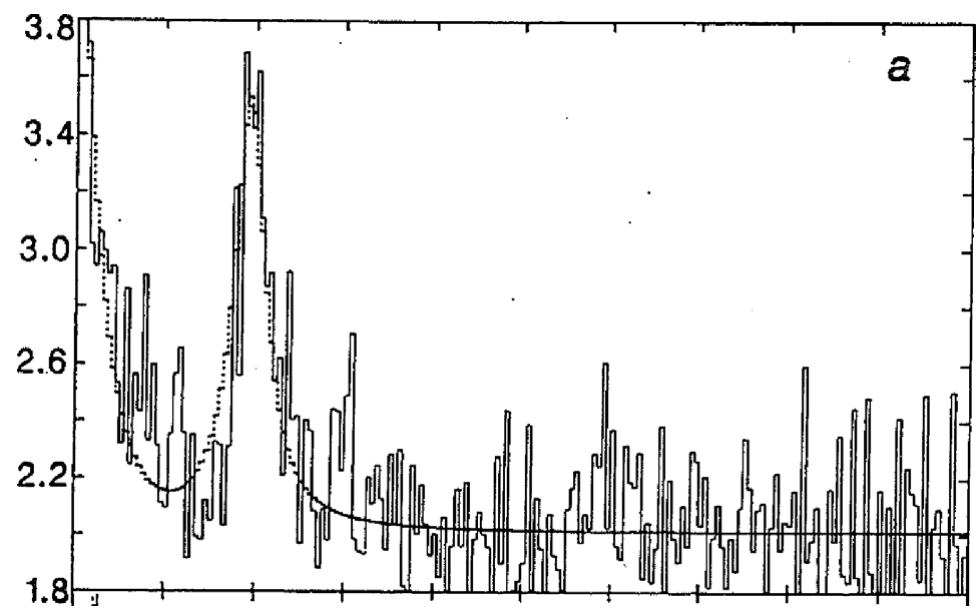

Figure 2. QPOs in X-Ray Binaries; the horizontal axis displays a frequency range from 0 to $100 \mathrm{~Hz}$ (van der Klis et al. 1985).

in some white dwarfs. Later recognition, from X-Ray observations starting in the 1970s, of binary systems containing neutron stars and black holes produced a wide range of the very short-time-scale phenomena naturally associated with these even more compact objects.

At radio frequencies, where compact extra-galactic sources were principal objects of study, observations initially trailed optical progress by one or two decades, so it was only in 1968 that a review of their variability was commissioned and written by Kellerman \& Pauliny-Toth (1968). The key conclusions were that "until recently, it had been generally felt that, apart from occasional supernova explosions or fluctuations in variable stars detected in nearby galaxies, no significant changes either in the radio or in the optical luminosity of extragalactic objects could take place on a time-scale shorter than the lifetime of a human observer", and that "the unexpected discovery of remarkably intense fluctations in the radio and optical luminosities of a number of quasi-stellar radio sources ... suggests that the previous conceptions of the processes involved in the generation of strong radio extragalactic radio sources ... were unjustified". Even more remarkably, just as the review was being written, it was upstaged by galactic radio sources, which generated a note added in press: "The discovery of an unexpected and entirely new class of variable radio source, announced in February 1968, has produced an unprecedented flurry of activity and publications". This was of course the announcement of the serendipitous discovery of pulsars (Hewish et al. 1969), found with a new radio telescope specifically constructed to search for rapid interplanetary scintillation which thus had the ability also to explore higher pulsation or rotation frequencies. In contrast to the 71-second modulation of DQ Her, which involved a white dwarf and caused only a short-lived gasp, the pulsars provided the first evidence for the long-predicted neutron stars, and as a result have become permanent star attractions. Other unexpected discoveries in this field were millisecond binary pulsars, of which several are now known, and planets in orbit around a millisecond pulsar, which was an example of a system so unexpected as to require almost a paradigm shift of interpretation.

Historically, observations at high energies gave strikingly unexpected results, with the first variable extra-solar X-Ray source, Sco X-1, found in 1962, the first X-Ray transients found in the late 1960s, and TeV gamma-ray flares from the blazar Markarian 501 found independently in 1997 by several ground-based Cherenkov telescopes (see Fig. 3). The 


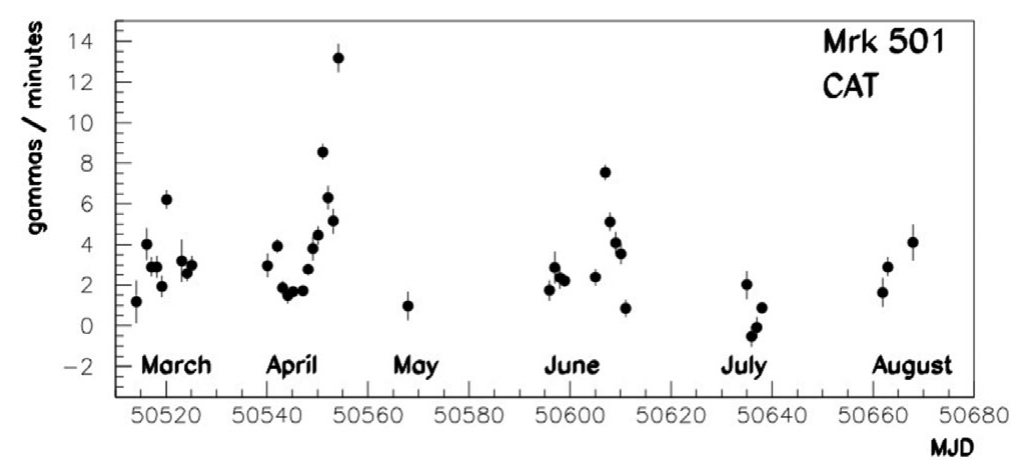

Figure 3. Gamma-ray flares in Markarian 501 (Protheroe et al. 1997).

first purely X-ray pulsar to be detected was Centaurus X-3, observed with a sounding rocket in 1967, but its 4.8-second periodicity was only found in 1971 with the Uhuru satellite - though by that time the Crab pulsar was already known to have pulsed XRays.

\section{Later Developments}

There is space-time here to discuss only a couple of the many unanticipated observational results that have created entire fields of research. Among degenerate stars, where the shortest time-scale variations are expected, highly stable rotation periods from seconds down to milliseconds were found among the neutron stars, down to $\sim 20$ seconds

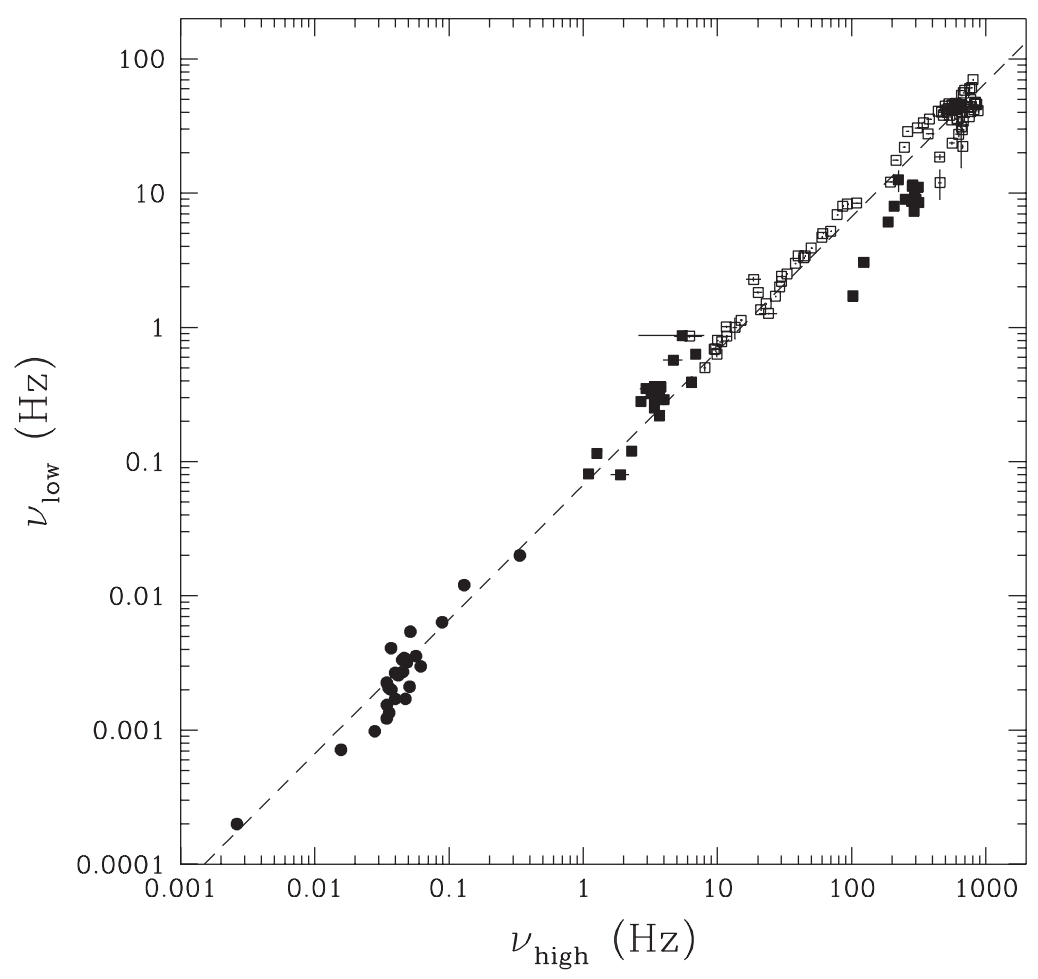

Figure 4. QPOs in Cataclysmic variables and X-Ray Binaries (Warner \& Woudt 2004). 

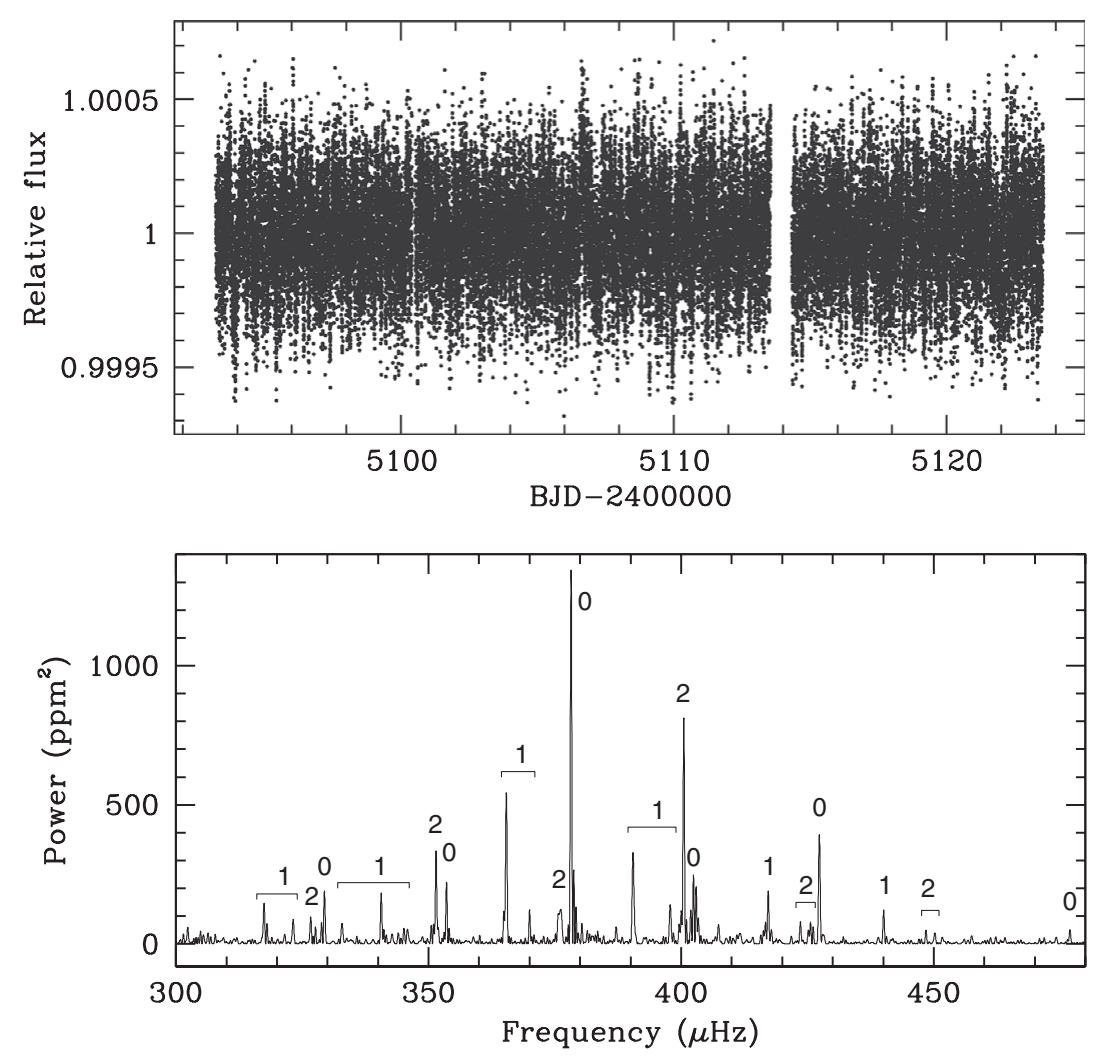

Figure 5. Solar-like oscillations in a red giant observed by KePLER (Di Mauro et al. 2011).

among white dwarfs, plus non-radial pulsations typically $\sim 100-1000$ seconds in some of them; but by searching, several kinds of quasi-periodic oscillations (QPOs) were discovered, including among black hole binaries. They were discovered first in cataclysmic variables (Warner \& Robinson 1972) and later in X-Ray binaries (van der Klis et al. 1985; see also Fig. 3). Currently these are probably the least well understood of the rapid phenomena that occur among the close binaries that contain accretion discs. In particular, the high-frequency and low-frequency luminosity oscillations (Fig. 2) show similar behaviour in all sources - the relevant physics to connect these disparate sources is not known (Warner 2006). Some binge thinking is required here.

During the past year results from the Kepler (2011) satellite, expected through asteroseismology to confirm and extend knowledge of non-radial acoustic pulsations in main sequence stars, and perhaps even to detect gravity-mode oscillations in red giants, have created a major new industry. Red giants turn out to rival dwarfs in the richness of their oscillations - this is now the era of ensemble asteroseismology: of 16500 giants observed in the KEPLER field in the first month $71 \%$ had oscillation spectra that could be analysed for period spacings. From the gravity-mode periods a clear distinction is obtained between those giants that are purely hydrogen shell-burning (period spacings $\sim 50$ seconds) and those that are in addition helium core-burning (spacings $\sim 100-300$ seconds) (Bedding et al. 2011). And there is even an unexpected bonus beyond this remarkable result: excess power at low frequencies in the Fourier transforms of $\sim 1000$ red giants is attributable to surface granulation - this is the first direct detection of the effects of surface convection in giant stars (Mathur et al. 2011). 
B. Warner

During this conference surveys that have already begun, or will be starting within a few years, are described in detail. In addition to these there is the proposed SUPERWASP Variable star survey, which should contain $\sim 800000$ periodic variables - and a possible SUPERWASP all-sky survey to record $\sim 1$ billion objects every 10 seconds over the whole sky, which will be rather data-intensive, generating $\sim 100$ Tb per day (Norton 2011).

These are all surveys for which we have high expectations of getting unexpected results; there are many smaller surveys and targeted observations which may unexpectedly find unexpected results. We have still to find evidence for some theoretic objects such as quark stars, quark novae, black stars, Thorne- $\dot{Z}$ ytkow objects, whose discovery, however, may simply be relegated by theoreticians as 'expected'. What may be required to excite them will be the unexpected unexpected. So, as the subject evolves over the next few years, expect the unexpected-be prepared for it.

\section{Acknowledgements}

My research is supported by the University of Cape Town and the National Research Foundation.

\section{References}

Bedding, T. R., et al. 2011, Nature, 471, 608.

Burke, B. F. \& Franklin, K. L. 1955, J. Geophys. Res., 60, 213

Di Mauro, M. P., et al. 2011, MNRAS, 415, 3783

Eyer, L. \& Cuypers, J. 2000, ASP Conf. Ser., 203, 71

Fabian, A. C. 2010, in: M. de Rond \& I. Morley (eds.), Serendipity: Fortune and the Prepared Mind (Cambridge University Press), p. 73

Franklin, K. L. 1959, AJ, 64, 37

Goldreich, P. \& Lynden-Bell, D. 1969, ApJ, 156, 59

Hewish, A., et al. 1969, Nature, 224, 472

Kellerman, K. I. \& Pauliny-Toth, I. I. K. 1968, Ann. Rev. Astr. Astrophys., 6, 417

Kepler 2011, http://kepler.nasa.gov/

Linnell, A. P. 1949, Sky \&3 Tel., 8, 166

Mathur, S., et al. 2011, arXiv:1109.1194

Motizuki, Y., et al. 2009, arXiv:0902.3446

Norton, A. 2011, http://www.star.le.ac.uk/conf50/talks/S01_1215_Andy_Norton.pdf

Protheroe, R. J., et al. 1997, arXiv:astro-ph/9710118

Ronan, C. A. 1994, The Shorter Science and Civilization in China, Cambridge University Press van der Klis, M., et al. 1985, Nature, 316, 225

Walker, M. W. 1956, ApJ, 123, 68

Warner, B. 2006, PASP, 116, 115

Warner, B., Robinson E. L. 1972, Nature Phys. Sci., 239, 2

Warner, B. \& Woudt, P. A. 2004, IAU Colloq., 315, p. 87 